\title{
Emprendimiento endógeno en comunidades indígenas del suroccidente colombiano
}

\author{
Héctor Fernando Rueda-Rodríguez ${ }^{1}$ \\ Universidad del Valle \\ hector.fernando.rueda@correounivalle.edu.co \\ Carlos Hernán González-Campo ${ }^{2}$ \\ Universidad del Valle \\ carlosh.gonzalez@correounivalle.edu.co
}

Fecha de recepción: 17 de octubre de 2019

Fecha de aprobación: 10 de marzo de 2021

Fecha de publicación: 06 de agosto de 2021

Cómo citar este artículo / To reference this article / Comment citer cet article / Para citar este artigo:

Rueda-Rodríguez, H. F.; González-Campo, C. H. (2021). Emprendimiento endógeno en comunidades indígenas del suroccidente colombiano. Revista Escuela de Administración de Negocios, (90), 85-100. DOI: https://doi.org/10.21158/01208160.n90.2021.2979

\section{Resumen}

El objetivo de este artículo es caracterizar aspectos relacionados con el emprendimiento endógeno en comunidades indígenas del suroccidente colombiano, desde la comprensión de sus tradiciones y costumbres, entre otros elementos. En la metodología se recogen datos que se analizan con métodos cualitativos desde la reunión de historias, los encuentros de saberes y las entrevistas realizadas en profundidad, con el fin de entender las vocaciones productivas de la comunidad y así caracterizar las dinámicas del emprendimiento endógeno en la comunidad Emberá-Chamí del resguardo Dachi Drua, ubicado en el municipio de Tuluá, Colombia. A partir del reconocimiento de la estrecha relación entre el emprendimiento endógeno, en el que se evidencia, a nivel teórico, una oportunidad de desarrollo conceptual, con los conceptos de identidad colectiva y desarrollo endógeno, los hallazgos presentan algunas estrategias de acción necesarias para afrontar retos relacionados con el desarrollo endógeno en la sociedad actual, con las particularidades del contexto colombiano. El aporte principal de este estudio está relacionado con el entendimiento y la caracterización de aspectos tanto históricos como sociales vividos por las comunidades indígenas que, a pesar de los cambios ideológicos, culturales y físicos, se han mantenido como parte fundamental de las raíces culturales del país en la construcción de una identidad colectiva que permea a todos los individuos, pues hacen parte de una configuración multicultural que se debe preservar.

Palabras clave: emprendimiento endógeno; emprendimiento en comunidades indígenas; comunidad Emberá-Chamí; identidad colectiva; desarrollo endógeno; vocaciones productivas; perfiles productivos.

\footnotetext{
Ingeniero industrial - Universidad INCCA de Colombia. Magíster en Ciencias de la Organización - Universidad del Valle. Estudiante del doctorado en Ciencias Ambientales - Universidad del Valle. ORCID: https://orcid.org/0000-0001-7998-0328

2 Economista - Universidad del Valle. Magíster en Ciencias de la Organización - Universidad del Valle. Doctor en Administración Universidad EAFIT. ORCID: https://orcid.org/0000-0003-2120-8209
} 


\title{
Endogenous entrepreneurship in indigenous communities of south-western Colombia
}

\begin{abstract}
The aim of this article is to characterize aspects that are related to endogenous entrepreneurship in indigenous communities of south-western Colombia, from the understanding of their traditions and customs, among other elements. In the methodology, we collected data to be analyzed with qualitative methods from the collection of stories, knowledge meetings, and in-depth interviews, in order to understand the productive vocations of the community and thus characterize the dynamics of endogenous entrepreneurship in the Emberá-Chamí community of the Dachi Drua reservation, located in the municipality of Tuluá, Colombia. Based on the recognition of the close relationship among endogenous entrepreneurship, in which there is evidence, at a theoretical level, of an opportunity for conceptual development, with the concepts of collective identity and endogenous development, the findings present some strategies for action that are necessary to face challenges related to endogenous development in today's society, with the particularities of the Colombian context. The main contribution of this study is related to the understanding and characterization of historical and social aspects experienced by indigenous communities that, despite ideological, cultural and physical changes, have remained as a fundamental part of the country's cultural roots in the construction of a collective identity that permeates all individuals, as they are part of a multicultural configuration that must be preserved.
\end{abstract}

Keywords: endogenous entrepreneurship; entrepreneurship in indigenous communities; Emberá-Chamí community; collective identity; endogenous development; productive vocations; productive profiles.

\section{Empreendedorismo endógeno em comunidades indígenas no sudoeste da Colômbia}

\section{Resumo}

O objetivo deste artigo é caracterizar aspectos relacionados ao empreendedorismo endógeno em comunidades indígenas do sudoeste da Colômbia, a partir da compreensão de suas tradições e costumes, entre outros elementos. Na metodologia coleta-se dados que são analisados com métodos qualitativos a partir de coleta de histórias, encontros de conhecimento e entrevistas realizadas em profundidade, a fim de compreender as vocações produtivas da comunidade e, assim, caracterizar a dinâmica do empreendedorismo endógeno na comunidade Emberá-Chamí da reserva Dachi Drua, localizada no município de Tuluá, Colômbia. A partir do reconhecimento da estreita relação entre o empreendedorismo endógeno, que mostra, teoricamente, uma oportunidade de desenvolvimento conceitual, com os conceitos de identidade coletiva e desenvolvimento endógeno, os resultados apresentam algumas estratégias de ação necessárias para enfrentar os desafios relacionados ao desenvolvimento endógeno na sociedade atual, com as particularidades do contexto colombiano. A principal contribuição deste estudo está relacionada à compreensão e caracterização dos aspectos históricos e sociais vivenciados pelas comunidades indígenas que, apesar das mudanças ideológicas, culturais e físicas, permaneceram como parte fundamental das raízes culturais do país na construção de uma identidade coletiva que permeia todos os indivíduos, pois fazem parte de uma configuração multicultural que deve ser preservada. 
Palavras-chave: empreendedorismo endógeno; empreendedorismo em comunidades indígenas; comunidade Emberá-Chamí; identidade coletiva; desenvolvimento endógeno; vocações produtivas; perfis produtivos

\section{Entrepreneuriat endogène des communautés autochtones du sud-ouest de la Colombie}

\section{Résumé}

L'objectif de cet article est de définir les aspects liés à l'entrepreneuriat endogène des communautés indigènes du sud-ouest de la Colombie, à partir notamment de la compréhension de leurs coutumes et traditions. La méthodologie utilisée recueillera des données qui seront ensuite analysées via des méthodes qualitatives à partir d'entretiens approfondis devant recueillir savoirs et connaissances afin de comprendre les vocations productives de la communauté et définir la dynamique de l'entrepreneuriat endogène dans la communauté Emberá-Chamí du Dachi, de la réserve de Drua, située dans la municipalité de Tuluá, en Colombie. Reconnaissant des relations étroites entre l'entrepreneuriat endogène offrant théoriquement des opportunités de développement conceptuel au travers des concepts d'identité collective et de développement endogène, les résultats de l'étude présenteront certaines stratégies d'action nécessaires pour faire face aux défis liés au développement endogène dans la société actuelle, et tenant compte des particularités du contexte colombien. La contribution principale de cette étude est liée à la compréhension et la caractérisation des aspects historiques et sociaux vécus par les communautés autochtones qui, malgré des changements idéologiques, culturels et physiques, sont toujours un élément fondamental des racines culturelles du pays pour la construction d'une identité collective imprégnant tous les individus.

Mots-clés: entrepreneuriat endogène; esprit d'entreprise dans les communautés autochtones; communauté Emberá-Chamí; identité collective; développement endogène; vocations productives; profils productifs. 


\section{Introducción}

$\mathrm{E}$ desarrollo endógeno se genera por medio del diálogo, la planificación y el entendimiento de los valores culturales, los saberes y las capacidades internas de las comunidades (de Franco, 2003). Lo anterior implica la generación de emprendimientos endógenos, el fortalecimiento y la organización comunitaria alrededor sistemas socioeconómicos articulados con diferentes ontologías e instituciones sociales y dependientes de los ecosistemas (Aponte y Álvarez, 2017; Ayres, van den Bergh y Gowdy, 2001; Leff, 2016). Así mismo, el emprendimiento endógeno en las comunidades indígenas, con miras a su sustentabilidad, se alcanza por sus vocaciones productivas y habilidades para la vida, es decir, todas las actividades realizadas con el propósito de lograr su manutención, lo cual se orienta, esencialmente, a la producción agrícola, la producción artesanal y el trabajo informal (Rojas, 2012).

También se evidencian referentes en la literatura de publicaciones sobre el emprendimiento social, como, por ejemplo, los planteados por GuzmánVásquez y Trujillo-Dávila (2008), otros más recientes sobre emprendimiento rural como los publicados por Marques et al. (2019), Peralta (2019) y Polbitsyn (2019), o sobre procesos de innovación social con comunidades propuestos, entre otros, por Mtika (2013) o Shin (2016). Lo anterior se enmarca en la búsqueda de una sustentabilidad mediante la creación de valor tanto económico como social, lo que propone un cambio de la visión netamente económica (Altieri y Toledo, 2011; Arroyave-Puerta y MarulandaValencia, 2019).

En este contexto, un factor fundamental en el estudio de las comunidades indígenas que en la actualidad perviven en Colombia es la identidad. Esta denota aspectos ancestrales únicos que se han deconstruido y reconstruido con el paso del tiempo (Heffes, 2012), así como está determinada por aspectos puntuales de cada contexto en el que se constituye. La identidad colectiva está relacionada conceptualmente con determinantes del sujeto que se conectan a su comunidad ampliada desde lo cognitivo, lo emocional o desde lo moral, lo cual surge de interrelaciones con audiencias diferentes y proporciona categorías que le permiten al individuo dividirse y darle sentido al mundo social (Polletta y Jasper, 2001). Desde un enfoque discursivo, se construye o se desarrolla mediante el reconocimiento de un determinado origen compartido o de características comunes entre personas, o también puede estar asociada a compartir ciertos ideales asumidos por un grupo de personas (Hall y du Gay, 2003).

En la comunidad estudiada el proceso de construcción de la identidad ha estado mediado por aspectos tanto históricos como recientes, representados en los cambios culturales y la apropiación de nuevas necesidades y costumbres, debido a un proceso de reubicación y nuevas formas de acomodación y asentamiento que los indígenas han vivenciado por diferentes factores estructurales.

A partir de lo anterior, este artículo pretende caracterizar aspectos del emprendimiento endógeno en las comunidades indígenas mediante la comprensión de aspectos socioeconómicos, tradiciones, costumbres y demás elementos que los identifican como miembros activos del resguardo indígena Emberá-Chamí Dachi Drua en el Valle del Cauca, Colombia. En la primera parte se incluyen los aspectos metodológicos de la investigación. En la segunda se presenta un marco teórico sobre emprendimiento endógeno que incluye un estado del arte con referentes de los últimos años. Finalmente, se presentan de forma descriptiva los principales hallazgos relacionados con el tema, los cuales se complementan con algunas ideas presentadas a modo de discusión y de conclusión. 
Los emprendimientos endógenos se analizan desde diferentes perspectivas y en distintos contextos. En la indagación se constata que, hasta el momento, no eran evidentes estudios similares, razón por la cual entender cómo las dinámicas del desarrollo endógeno y los elementos de la identidad colectiva influyen en la formulación de proyectos de emprendimiento endógeno en comunidades como la estudiada es el principal aporte de este artículo.

\section{Metodología}

$\mathrm{E}$ ntre los objetivos de la investigación se encuentran: a) caracterizar los principales aspectos socioeconómicos y demográficos de la comunidad, b) evidenciar las tradiciones y costumbres que permiten obtener emprendimientos endógenos en la comunidad estudiada, y c) evidenciar la relación de los participantes con aspectos del desarrollo económico en sus actividades productivas.

En este contexto, para esta investigación exploratoria se utilizan técnicas cualitativas en el análisis de la información compilada mediante: a) entrevistas en profundidad (Spradley, 1979), en busca de entender los procesos económicos, las motivaciones y los elementos de la identidad cultural; b) una cartografía social para mapear las comunidades e identificar las zonas de importancia y oportunidades de mejora; y c) se complementa con una encuesta poblacional de la comunidad.

En el abordaje metodológico se incluye un encuentro de saberes desde el que se compila la información y se caracterizan aspectos relacionados con las formas de producción y la identidad cultural de la comunidad. Además, se identifican posibles vocaciones productivas utilizando como referentes los siete ejes planteados por la Organización Regional de Indígenas del Valle del Cauca (Orivac):
«Territorio, economía y desarrollo propio; cultura y educación; gobernabilidad y jurisdicción; familia, género y generación; derechos humanos; salud y espiritualidad» (2012, p. 7). Esto con el fin de que, desde el respeto de su cosmogonía, se prioricen las principales problemáticas sociales y posibilidades productivas con respeto por el medio ambiente y la herencia cultural de estas comunidades.

Antes de presentar la revisión teórica sobre el emprendimiento endógeno y su relación con los conceptos de identidad colectiva y desarrollo endógeno, se procede con la delimitación del concepto organización indígena, entendida esta como las comunidades indígenas ubicadas en zona rural de la geografía nacional, organizadas y reconocidas como cabildos, resguardos y territorios. En el alcance de esta investigación, las comunidades indígenas del Valle del Cauca, desde el Decreto 1088 de 1993, están representadas por la Organización Regional Indígena del Valle del Cauca (Orivac) y la Asociación de Cabildos Indígenas del Valle-Región Pacífico (ACIVARP), fundadas en 1989 y 1997, respectivamente. Lo anterior evidencia la reciente conformación de las organizaciones indígenas por bloques de territorios. 


\section{Marco teórico}

$\mathrm{E}$ la revisión de bibliografía se evidencia que es escasa la literatura sobre emprendimiento endógeno y es más común abordar el emprendimiento social o local (Bargsted, 2013; Global Entrepreneurhip Monitor, 2016). La categoría de endógeno le da la cualidad al emprendimiento de ser desarrollado desde los saberes ancestrales y en concordancia con la cosmogonía de los pueblos originarios o indígenas (Ospina-Ramírez, 2018). En este mismo sentido, el concepto de desarrollo endógeno relaciona las categorías de lo social y lo ambiental con el desarrollo económico, a partir de la necesidad de una relación sostenible entre la economía y el medio ambiente en comunidades en situación de vulnerabilidad (Chirinos-Araque y Pérez-Peralta, 2016). Es entonces relevante reconocer que los actores locales, haciendo uso de los recursos, las instituciones, los conocimientos y las estrategias, benefician a la población a la vez que refuerzan las habilidades de integrarse con el medio ambiente (Rist, 2005).

En el contexto del desarrollo endógeno, el emprendimiento implica la construcción de proyectos productivos con la participación de la comunidad no solo operativa, sino representada en su cosmogonía e identidad cultural (OspinaRamírez,2018).En la revisión realizada se evidencia que los estudios existentes sobre emprendimiento indígena están orientados al estudio de la identidad, al examinar la relación entre la identidad indígena, la identidad colectiva y los perfiles productivos.

El otro concepto relevante en esta investigación es la identidad colectiva, en su relación con el emprendimiento o cualquier nivel que implique lo social. Es una categoría que se construye a partir de referentes comunes que pueden partir de la cultura de las comunidades. En este sentido, las ontologías se relacionan con las vivencias de las comunidades, su relación con otros y con los recursos disponibles en la zona (Hall y du Gay, 2003).
De esta forma, el reconocimiento de los rasgos distintivos que posicionan a los sujetos como seres con visiones y posturas con respecto a la vida cotidiana requiere de procesos de concientización que se entienden como una acción cultural para el cambio social (Freire, 1974). Con miras a esta orientación es posible plantear que la concientización es un proceso dialógico que le permite a los sujetos tener como referencia la historia de su cultura para así reivindicar sus tradiciones, sus prácticas, sus actos emprendedores y sus formas de percibir la realidad. Así mismo, plantear que el sujeto reconoce la comunidad como un asunto que también está en constante construcción y cambio, y que no es un proceso dado, sino que se modifica dependiendo de las particularidades del contexto.

La comunidad es una construcción social que se desarrolla en el tiempo a partir de la interacción con los otros (Torres, 2013; Vidal y Pol, 2004). En el caso de las comunidades indígenas, los rasgos étnicos son un factor fundamental para configurar lazos de fraternidad entre los sujetos que las conforman. A pesar de las luchas y las resistencias que han vivenciado, en las comunidades indígenas aún se conservan tradiciones propias de su cultura. Aun en un contexto de modernidad y de una fuerte tendencia a las costumbres occidentales, las comunidades indígenas luchan por preservar sus ancestrales costumbres, pero, pese a su resistencia, el individualismo propio de la civilización occidental, como política de Estado, condiciona la forma en la que se relacionan con el territorio y con los otros miembros de la comunidad (Sennett, 2007).

El territorio es también un factor que va a demarcar aspectos de identidad, teniendo en cuenta que se generan mecanismos de resistencia, permanencia y preservación de las culturas étnicas; además, se construyen lazos de hermandad y una simbología única que es valorada por las comunidades 
indígenas. La conservación de los territorios ancestrales y los recursos naturales son también sus objetivos para restablecer una conexión con su patrimonio (Peredo et al., 2004).

Así, la identidad colectiva se mantiene alrededor del territorio y su significado, pero esta se ha visto transgredida por múltiples factores estructurales, como, por ejemplo, la pobreza, la vulnerabilidad y la violencia arraigada en conflictos por la tenencia de tierras (Berkes y Adhikari, 2006), lo que ha redireccionado algunas prácticas tradicionales $\mathrm{y}$ ancestrales de estas poblaciones. En este sentido, la pérdida de las tradiciones culturales de las comunidades indígenas es consecuencia de fenómenos externos a la comunidad; aun así, las comunidades siguen en defensa de sus concepciones cosmogónicas alrededor del territorio, el reconocimiento a la diversidad cultural y el desarrollo local, para lo cual mantienen una postura de diálogo coherente y respetuoso con otras lógicas culturales (Zapata, 2010).

La configuración territorial permite establecer vínculos alrededor de recursos bióticos y otros miembros de la comunidad, lo que trae armonía entre los pueblos (Salazar, 2016) y tiene una connotación simbólica del tipo espiritual; de ahí que existan recursos que no deben ser explotados por poseer una connotación sagrada o por vincular la cosmogonía de la comunidad (Haesbaert, 2013).
Las comunidades indígenas desarrollan mecanismos de organización comunitaria a partir de la identificación de elementos culturales compartidos y un pasado en común (Fals-Borda, 2016). Esto les permite identificar los problemas que comparten con los demás miembros de la comunidad y generar un diálogo alrededor de la identificación de los recursos disponibles, lo que incluye saberes ancestrales y la planificación de las acciones a seguir. Al mismo tiempo, permite caracterizar aspectos propios del desarrollo de distintas actividades, entre ellas las determinadas por procesos de emprendimiento.

El proceso de organización para desarrollar una actividad productiva es variable y depende de la actividad que se va a desarrollar, el tiempo con que se cuenta para su ejecución, los recursos disponibles y su importancia relativa o la expectativa que genere. Esta última está condicionada por los sujetosactores, ya que, dependiendo de su condición, su rol en la sociedad o en la comunidad, se les da mayor importancia a algunas actividades.

Con lo anterior, se reconoce la estrecha relación entre el emprendimiento endógeno, en el que se evidencia, a nivel teórico, una oportunidad de desarrollo conceptual con los conceptos de identidad colectiva y desarrollo endógeno.

\section{Resultados y discusión}

\subsection{La organización indígena e identidad colectiva}

La Páez representa una de las comunidades indígenas más importantes en Colombia. De acuerdo con el DANE (2005), pertenecen a ella 118845 personas, lo que corresponde al $16,93 \%$ de la población indígena en el país. Esta comunidad, en busca de adaptarse a los cambios y las transformaciones que apremiaron en el siglo $\mathrm{XX}$, emprendió procesos organizativos desde el reconocimiento de su territorio, de manera que ha logrado pervivir en medio de las dificultades de la modernidad (Rojas, 2012).

Desde una perspectiva más amplia, es desde las comunidades indígenas que se construye la identidad colectiva y las formas de organización, 
con base en el fortalecimiento interno, en medio de los desplazamientos y la expropiación de la tierra que han vivido a causa de múltiples factores, asociados, principalmente, a políticas de Estado y al modelo social y económico capitalista.

Al respecto, Feldman-Bianco, Domenech y Sanjurjo (2020) señalan: «Frente a un escenario marcado por un capitalismo corporativo destructivo, estos desplazamientos evidencian el surgimiento de nuevas lógicas de producción, desigualdades, fronteras sociales y contingentes de desposeídos» (p. 8). Según los estudios de Rojas (2012), se identifica que el único movimiento social en el siglo XX que alcanzó un protagonismo político en el país fue el de los indígenas, lo cual se logró gracias a la lucha constante por sus derechos. De esta manera, consiguieron que se estipulen leyes de protección y preservación de su cultura, como, por ejemplo, el Auto 04 de 2009.

De esta lucha fueron pioneros los indígenas paeces del departamento del Cauca, en los inicios del siglo XX. Esta lucha fue conocida con el nombre de la «Quintinada» debido al reconocimiento de su líder y representante Manuel Quintín Lame, quien logró que en 1971 se constituyera el Consejo Regional de Indígenas del Cauca (CRIC) (Rojas, 2012).

Se evidencia que la comunidad Emberá-Chamí, como muchas poblaciones indígenas en el país, ha sido desplazada de sus territorios, incluso fragmentada y recompuesta por mandatos del Estado; es decir, no hay una comunidad de base $\mathrm{u}$ originaria, sino que se ha compuesto por la unión de personas de distintos resguardos de otros departamentos. Aunque la resistencia es un factor que se identifica en muchas comunidades indígenas, aspectos tales como el esparcimiento, el cambio de territorio y la reubicación han sido elementos que tienden a transgredir la identidad colectiva de las comunidades y, por ende, sus tradiciones, lo que ha generado transformaciones en sus dinámicas internas, e incluso pérdida de costumbres ancestrales.
Estos efectos son descritos también en comunidades maorí de Nueva Zelanda, en las que el desafío de hacer cumplir los límites frente a la colonización -especialmente los efectos de la guerra, la enfermedad, el hambre, el aislamiento económico, el desplazamiento y la reubicación一, la explotación de recursos y el desarrollo urbano las ha forzado a competir, de modo que se transgrede un límite proscrito en el que las relaciones internas y las externas inherentes se ven amenazadas (Tapsell y Woods, 2008).

La comunidad Emberá-Chamí vivió los procesos de colonización y la implementación de nuevas prácticas y formas de vida; respecto a lo cual se señala:

La estrecha relación del pueblo Emberá-Chamí con la tierra empezó a desintegrarse con el inicio del proceso de conquista española y que desembocó en el abandono forzado de las tierras con las que habían creado esa relación y su reemplazó por territorios de selva húmeda, con suelo, clima y productividad diferente. Estas circunstancias redujeron la disponibilidad de los recursos que ofrecía la tierra y transformaron las dinámicas internas de las comunidades, incorporando la noción de propiedad no colectiva y con ésta, una nueva estructura de grupo. (Organización Nacional Indígena de Colombia [ONIC], 2018, p. 2)

Las formas de organización de las comunidades se han visto como procesos complejos y son transversales a la autoridad, la gobernabilidad y el crecimiento económico interno. Es así como la autoridad va a demarcar un orden central establecido, regido por normativas que son aceptadas y legitimadas como una jurisdicción indígena inamovible, es decir, están arraigadas y naturalizadas en la vida cotidiana de los individuos que componen la comunidad. Al ser una normativa respetada y aplicada en todos los resguardos indígenas, va a simbolizar una estructura social que está compuesta por la cultura, la espiritualidad, la familia, la economía y el desarrollo propio. En este sentido, Rojas (2012) se refiere a la autoridad en las comunidades indígenas como «los pater- 
familias, que generalmente son quienes dirigen la comunidad y tienen una autoridad moral y una autoridad política. La autoridad política viene de lo moral» (p. 180).

De esta forma, si bien las comunidades indígenas han logrado estructurar un sistema político y social en la modernidad, también se dimensionan dificultades de gran magnitud que han transgredido su identidad y el bienestar colectivo. La lucha por mantener condiciones de vida dignas bajo el capitalismo que rige el modelo social y económico han llevado a establecer relaciones - en muchas ocasiones- asistenciales con los gobiernos locales y nacionales.

Aunque desde 1991 el Estado reconoció legalmente derechos a comunidades indígenas, en muchas partes del país, especialmente en el suroccidente colombiano, ha configurado relaciones asistenciales y de regulación con las comunidades indígenas, ya que los recursos destinados a estas no se ejecutan según un plan basado en la autonomía para agenciar su desarrollo económico.

En este contexto, una reflexión en las comunidades evidencia que la producción interna de tipo agrícola o artesanal no abastece de forma suficiente sus necesidades, lo que conduce a los individuos a emplearse en actividades informales. Las personas ejercen estas labores, principalmente, como trabajadores, constructores o jornaleros en fincas aledañas a su resguardo, en la mayoría de los casos (Rojas, 2012).

En otras palabras, se espera que la comunidad sea capaz de sobreviviry crecer de manera autosuficiente y endógena (Maillat, 1998), con actividades tradicionales o adquiridas recientemente; estas vocaciones van a aludir a rasgos culturales y de identidad, como, por ejemplo, la producción agrícola, tanto para la venta como para el consumo diario y la producción artesanal. Esta consiste en la producción de bolsos, collares y bisutería realizada a mano y con chaquiras, con materiales muy utilizados por mujeres y hombres que se dedican a esta labor; por ejemplo, se utilizan semillas de árboles en la elaboración de estas artesanías.
Cabe mencionar que el Gobierno colombiano, reconociendo las múltiples problemáticas que enfrentan las comunidades indígenas en materia ambiental, económica, social e institucional, ha adoptado la metodología de perfiles productivos para entender las características y vocaciones productivas ideales en un escenario de emprendimiento social o empresarial. El perfil productivo es definido por aquellos rasgos distintivos y las orientaciones productivas de una región que determinan las actividades económicas que pueden ser realizadas (Programa de las Naciones Unidas para el Desarrollo [PNUD], 2014).

Los perfiles productivos permiten identificar las potencialidades y debilidades, así como realizar un estudio del territorio y los elementos que pueden contribuir a la producción, a fin de que las comunidades logren alcanzar su propio desarrollo, con una economía sostenible y sólida, de tal modo que se adquiera una autonomía con respecto a la inversión y la toma de decisiones para su progreso interno.

Similar a la situación de la comunidad EmberáChamí del resguardo Dachi Drua es la de otras comunidades indígenas en el mundo (Khan, 2014), en las que requieren ayuda para superar su aislamiento y vulnerabilidad de manera sostenible, con estrategias que sean autosuficientes y las empoderen, a partir de su identidad cultural y productiva, a fin de enfrentarse a las dinámicas de la población general, por medio de la educación y la formación.

Las propuestas de emprendimiento empresarial y productivo dentro de las comunidades indígenas defienden la conservación de las tradiciones productivas y culturales, de ahí su carácter endógeno, el cual logra preservarse cuando se implementa con participación de la población implicada. Bajo los parámetros de la investigación participativa, el conocimiento y la acción cambian o transforman una realidad dada y facilitan la comprensión de esta resaltando la importancia del 
trabajo en equipo, la participación, la reflexión y la generación de un conocimiento desde el saber popular (Murillo, 2018).

Con respecto a este estudio, la investigación participativa permitió conocer y establecer metodologías de trabajo desde las necesidades de la comunidad, lo que representó una construcción conjunta de saberes y facilitó el aprovechamiento de las capacidades de la población para ser agentes de su desarrollo y crecimiento económico.

En la comunidad estudiada lograron identificar algunas actividades económicas que generan bienestar y pueden sustentar su desarrollo económico endógeno. Además, se reconocieron actividades con potencial para el futuro que se pueden implementar dentro de los territorios indígenas con miras a generar mayores oportunidades de desarrollo propio.

El aporte principal de este estudio está relacionado con el entendimiento y la caracterización de aspectos tanto históricos como sociales vividos por las comunidades indígenas que, a pesar de los cambios ideológicos, culturales y físicos, se han mantenido como parte fundamental de las raíces culturales del país en la construcción de una identidad colectiva que permea a todos los individuos, pues hacen parte de una configuración multicultural que se debe preservar.

\subsection{Aspectos relevantes para el desarrollo endógeno evidenciados y una propuesta de fortalecimiento de las vocaciones productivas}

El resguardo Dachi Drua cuenta con un total de 113 habitantes, 55 son mujeres y 58 son hombres. Los mayores grupos etarios están entre cero a cinco $-25,66 \%$ y seis a diez años - 16,81\%-, que representan el $42,47 \%$ respecto al total de la población; el rango de los 11 a los 25 años corresponde al 32,74 \%, es decir, al sumar estos rangos de edad, con una población de niños y adolescentes del $75,21 \%$ respecto a la población. Las personas entre los 26 y los 30 años componen el 9,73\%, jun- to con el adulto joven que oscila entre los 31 a 40 años, que representan el 6,19\% de habitantes en la edad más productiva. Los adultos que van desde los 41 a los 60 años representan el 5,3\% y los adultos mayores de 60 años en adelante es la población menos preponderante con un 3,54\%. En términos generales, la población en edad de trabajar va de los 16 a los 60 años y corresponde al 41,57\% del total de la población. En promedio, habitan 25 personas en cada vivienda y los grupos familiares se componen de cuatro a 12 personas.

En coherencia con la perspectiva metodológica del estudio, los hallazgos son presentados en los siete ejes planteados por la Orivac en el 2012. El primer eje es territorio y hace referencia a las áreas en donde habitan las comunidades y desarrollan actividades sociales, económicas y culturales. El segundo eje es economía y desarrollo propio, el cual se refiere a los procesos de producción, transformación, comercialización y consumo dentro del territorio. El tercer eje es cultura y educación, referido al conjunto de procesos que recogen el pasado, los antepasados y el presente de las comunidades indígenas, al igual que sus cosmogonías y los principios que los orientan.

El cuarto eje es gobernabilidad y jurisdicción, relacionado con las formas de gobernar de cada comunidad según su cultura a través de normas, formas de comportamiento y la relación con su entorno. El quinto eje es familia, género y generación y se refiere a la participación política de las mujeres en los distintos espacios de la vida social, a la concepción de familia y los roles según el género y la generación. El sexto eje es derechos humanos y hace referencia al conocimiento de la comunidad acerca de sus derechos y deberes como ciudadanos, así como al ordenamiento jurídico indígena. Finalmente, el séptimo eje es salud y espiritualidad, el cual se relaciona con el acceso al sistema de salud en Colombia y la calidad de vida.

En la tabla 1 se presentan las características evidenciadas para cada uno de los siete ejes propuestos por la Orivac (2012). 
Tabla 1. Caracterización por eje

\begin{tabular}{|c|c|}
\hline Eje & Hallazgo \\
\hline $\begin{array}{l}\text { Eje 1. } \\
\text { Territorio }\end{array}$ & $\begin{array}{l}\text { El resguardo está compuesto por } 136 \text { hectáreas en las que los principales cultivos son plátano, café, } \\
\text { frijol, maíz y yuca. Dentro del resguardo existen zonas que potencialmente se pueden enfocar en } \\
\text { actividades productivas, como, por ejemplo, un lago, en el que se cultivan peces que son vendidos } \\
\text { a los campesinos de la región. En la realización del mapa del futuro con respecto al territorio, los } \\
\text { participantes manifestaron que un acueducto reparado y estable puede nutrir de agua el lago en el que } \\
\text { podrían cultivar peces. También visionan la siembra de caña y la implementación de un trapiche para } \\
\text { posicionarse como productores de panela en la localidad, lo cual se ve como una potencial vocación } \\
\text { productiva. }\end{array}$ \\
\hline $\begin{array}{l}\text { Eje 2. Economía y } \\
\text { desarrollo propio }\end{array}$ & $\begin{array}{l}\text { De acuerdo con la ocupación de los miembros del resguardo, se encontró que el } 76,92 \% \text { se desempeñan } \\
\text { en la agricultura, el 12,82 \% son empleadas en oficios varios y el } 2,56 \% \text { se desempeñan como madres } \\
\text { comunitarias, empleadas domésticas, modistas y profesores. La actividad agrícola se centra en la } \\
\text { siembra de café y plátano, desarrollada en las tierras de cada familia que conforma el resguardo. }\end{array}$ \\
\hline $\begin{array}{c}\text { Eje } 3 . \\
\text { Cultura y educación }\end{array}$ & $\begin{array}{l}\text { El } 20,35 \% \text { de la población está en proceso de formación, representado por niñas y niños. De la } \\
\text { población entre los } 16 \text { y } 40 \text { años - } 36,98 \% \text {, el } 17,70 \% \text { está terminando el bachillerato. Respecto } \\
\text { a la alfabetización en menores, se encontró que el } 60,18 \% \text { sabe leer y escribir, es decir, un poco más } \\
\text { del } 50 \% \text { de la población, la cual, en su mayoría, corresponde a niños, niñas y adolescentes que asisten } \\
\text { a la escuela. En relación con las necesidades sentidas por los indígenas en cultura y educación han } \\
\text { surgido algunas prácticas por la influencia de la cultura dominante que han generado sentimientos de } \\
\text { insatisfacción y carencia. Por ejemplo, en lo concerniente al vestuario, que ha sido modificado con el } \\
\text { pasar del tiempo, se han dejado de lado sus trajes tradicionales por las influencias y el rechazo de las } \\
\text { comunidades que viven a su alrededor. Como lo menciona uno de los integrantes de la comunidad } \\
\text { Emberá-Chamí: «Cuando nosotros llegamos a esta región hace } 20 \text { años, las mujeres utilizaban su traje } \\
\text { típico, pero como estamos viviendo alrededor de campesinos y hay una escuela, entonces en la escuela } \\
\text { les decían que eran de mil colores, que porque tenían colores extravagantes y pues ya les fue dando } \\
\text { pena y poco a poco se fueron acostumbrando a la ropa común, ya no ropa hecha por ellos sino ropa que } \\
\text { compran en el pueblo de color más sencillo. Prendas hechas por nosotros mismos ya no hay». } \\
\text { La reducción del territorio para la comunidad indígena ha generado impactos negativos en las } \\
\text { costumbres ancestrales, ya que ha permeado su cultura por la influencia de la comunidad campesina } \\
\text { que confluye en el mismo territorio. }\end{array}$ \\
\hline $\begin{array}{l}\text { Eje } 4 . \\
\text { Gobernabilidad y } \\
\text { jurisdicción }\end{array}$ & $\begin{array}{l}\text { Las normas y leyes regulan el comportamiento de la comunidad. Es deber del gobernador, como } \\
\text { máxima autoridad, servir de mediador en los conflictos y guía de comportamiento en los aspectos más } \\
\text { triviales de la convivencia. El gobernador es elegido por voto popular y como requisito debe ser un } \\
\text { miembro activo de la comunidad y tener entre diez y } 60 \text { años de edad. }\end{array}$ \\
\hline $\begin{array}{l}\text { Eje } 5 . \\
\text { Familia, género y } \\
\text { generación }\end{array}$ & $\begin{array}{l}\text { La comunidad Emberá-Chamí tiene una marcada división de las tareas dependiendo si se es hombre } \\
\text { o mujer. En este sentido, las mujeres se dedican a lo local y los hombres a lo social. Las tareas de las } \\
\text { mujeres involucran los oficios domésticos, la siembra y recolección de alimentos. La comunidad es un } \\
\text { patriarcado, ya que es el hombre el que ejerce cargos de poder político. }\end{array}$ \\
\hline $\begin{array}{c}\text { Eje } 6 . \\
\text { Derechos humanos }\end{array}$ & $\begin{array}{l}\text { La comunidad identifica los derechos hacia la correspondencia del Estado respecto a recursos y } \\
\text { asistencia para la comunidad. En este sentido se expresó: «En muchos resguardos o asentamientos, } \\
\text { muchos no conocen los derechos y por eso la ayuda del Estado es muy poca; porque no hay una persona } \\
\text { verdaderamente experta que pueda tocar puertas a la institución» (comunicación personal, integrante } \\
\text { de la comunidad Emberá-Chamí del municipio de Tuluá). }\end{array}$ \\
\hline $\begin{array}{l}\text { Eje } 7 . \\
\text { Salud }\end{array}$ & $\begin{array}{l}\text { La comunidad está cubierta por el sistema de salud nacional. Sin embargo, en el momento de enfrentar } \\
\text { la enfermedad, el } 58,8 \% \text { de los encuestados tiende a tratarlas en primera instancia dentro del resguardo, } \\
\text { acudiendo a la medicina tradicional, mientras que el } 41,2 \% \text { acuden directamente a centros de salud } \\
\text { como primera opción para atender cualquier problema de esta índole. La problemática principal de la } \\
\text { comunidad en lo que concierne a la salud es originada por la vivienda, que se encuentra en mal estado } \\
\text { y genera problemas de salubridad y convivencia por la falta de privacidad que violenta los límites de los } \\
\text { espacios individuales y personales. }\end{array}$ \\
\hline
\end{tabular}

Fuente. Elaboración propia. 
Así mismo, en lo que concierne a la identificación de las vocaciones productivas, uno de los aportes relevantes de este estudio se evidencia en que las artesanías propias del resguardo son factibles de ser producidas y comercializadas. En este sentido, se trata de una actividad cuyo saber lo posee la mayor parte de la comunidad, es decir, ocho de cada diez personas, y tienen la disponibilidad de replicar dicho aprendizaje, aunque para ello se deben fortalecer mecanismos de participación comunitaria que propicien la construcción colectiva de acciones y actividades que permitan ejercicios de planeación alrededor de las problemáticas más sentidas por la comunidad y los proyectos productivos con mayor potencial de ser desarrollados.

De acuerdo con lo encontrado en el estudio, se determinan dos programas con el fin de fortalecer las actividades económicas endógenas en la comunidad. El primero es el Programa de fortalecimiento de habilidades técnicas, en el que los conocimientos sobre la fabricación y realización de artesanías son propios de las comunidades indígenas y son útiles para promover la diversificación de estas mediante la implementación de buenas prácticas de producción, administración y comercialización de los productos. Esta actividad se puede desarrollar por medio de la capacitación y la apropiación de herramientas que permitan, desde un lenguaje sencillo, transmitir los elementos necesarios para que los individuos puedan vender sus artesanías a un precio justo en el que puedan incluir sus ganancias e inversión.

El segundo es el Programa de fortalecimiento de la participación y organización comunitaria, por el cual se pretende desarrollar capacidades de gestión en comunidades étnicas desde un reconocimiento de lo propio, la articulación con la institucionalidad y lo público, así como el diálogo intercultural, ya que es importante para adquirir capital social y nuevas formas de emprender la conexión con instituciones y empresas del sector en otros mercados.

En la tabla 2 se presentan, a modo de síntesis, los elementos principales de cada uno de los programas.

Tabla 2. Descripción de los programas propuestos

\begin{tabular}{|c|c|}
\hline Programa & Descripción \\
\hline $\begin{array}{l}\text { Programa de } \\
\text { fortalecimiento de } \\
\text { habilidades técnicas }\end{array}$ & $\begin{array}{l}\text { Este programa debe contar con los siguientes puntos. } \\
\text { - Diseño y desarrollo de productos en talleres artesanales. } \\
\text { - Fortalecimiento empresarial mediante capacitación, desarrollo de listas de precios, definición } \\
\text { de materia prima, costos de funcionamiento y costos totales en cada uno de los talleres } \\
\text { artesanales. } \\
\text { - Desarrollo de herramientas de comercialización y promoción para cada taller artesanal. } \\
\text { Participación en ferias, eventos de promoción y comercialización que se presenten a nivel } \\
\text { nacional e internacional para dar a conocer la cultura por medio de los productos que venden. }\end{array}$ \\
\hline $\begin{array}{l}\text { Programa de } \\
\text { fortalecimiento de } \\
\text { la participación y } \\
\text { organización comunitaria }\end{array}$ & $\begin{array}{l}\text { Este programa debe contar con los siguientes puntos. } \\
\text { - El empoderamiento de las comunidades a partir de sus propios núcleos culturales y } \\
\text { - } \text { lingüísticos. } \\
\text { El fortalecimiento de las capacidades de gestión autónoma para generar competencias } \\
\text { dirigidas al relacionamiento institucional en las comunidades étnicas. } \\
\text { - La generación de procesos de cohesión a partir de la gestión autónoma. } \\
\text { - La constitución de un semillero artesanal con los niños, las niñas y los adolescentes dentro } \\
\text { de las comunidades para que se realice la transmisión de saberes y así aprendan el arte y las } \\
\text { técnicas ancestrales de su comunidad, a fin de contribuir a la preservación de su identidad y } \\
\text { su cultura. }\end{array}$ \\
\hline
\end{tabular}

Fuente. Elaboración propia. 
Los programas de desarrollo endógeno fortalecen la economía de la comunidad y permiten consolidar los agentes de transformación social, en la medida en que reconocen y aprovechan las potencialidades del territorio y los saberes ancestrales expresados en capacidades productivas, de manera que aportan al desarrollo social y económico. Estos hallazgos coinciden con los encontrados por Gallagher y Lawrence en el 2012, quienes proponen que el emprendimiento indígena no va en contra de las identidades sociales de las comunidades; por el contrario, son un aspecto vital de las comunidades indígenas en el propósito de lograr sus sueños individuales y colectivos, así como de mantener y fortalecer su cultura, mientras utilizan mecanismos económicos como, por ejemplo, el espíritu empresarial, para ganarse la vida, retribuir a sus comunidades y trabajar hacia su autosuficiencia.

Los emprendimientos endógenos, generados desde la cosmogonía de las comunidades indígenas, aportan a la construcción de escenarios sociales y políticos que promueven la no violencia, aportan al fomento de la paz, la promoción y el reconocimiento de los derechos humanos, buscan favorecer el cuidado del medio ambiente, garantizar la seguridad alimentaria, reducir las desigualdades y aliviar la pobreza. De esta manera, también aportan al cumplimiento de los Objetivos de Desarrollo Sostenible (Organización de las Naciones Unidas [ONU], 2015) por parte de Colombia, en el compromiso adquirido con sus metas al 2030.

De igual forma, las dinámicas sociales $y$ económicas sustentables benefician directamente al sector privado al reducir los costos económicos asociados al fenómeno de la violencia (Guáqueta y Mantilla, 2005), lo que puede favorecer posibles alianzas estratégicas entre el sector empresarial y las comunidades indígenas.

\section{Resultados y discusión}

ـ investigación participativa permitió codesde las necesidades de la comunidad, lo que representa una construcción conjunta de saberes y el aprovechamiento de las capacidades de la población para ser agentes de su desarrollo y crecimiento económico. Con el trabajo en equipo, la comunidad identificó sus habilidades y capacidades a fin de generar emprendimientos endógenos que duren en el tiempo y conserven sus rasgos distintivos de identidad cultural como comunidad indígena.

En la comunidad se logran identificar algunas actividades económicas que generan bienestar y pueden sustentar su desarrollo económico endógeno, además de reconocer actividades con potencial para el futuro que se pueden implementar dentro de los territorios indígenas para generar mayores oportunidades de desarrollo propio.

Las comunidades indígenas en cualquier parte del territorio nacional presentan desafíos $y$ oportunidades similares, así como poseen recursos naturales muy valiosos, entre ellos bosques primarios, fauna y flora, recursos minerales, afluentes naturales y terrenos fértiles. $\mathrm{Al}$ respecto, Rojas (2012) señala cómo las actividades de manutención de las comunidades indígenas en Colombia están orientadas, esencialmente, hacia la producción agrícola y artesanal, o al trabajo informal. 
En este sentido, invertir en medios de producción, fortalecer las competencias laborales, buscar nuevos mercados para la comercialización de servicios y productos, siempre y cuando se gestionen con eficiencia los recursos naturales y se respete la cosmogonía e identidad colectiva de las comunidades, aporta en términos estratégicos al desarrollo sustentable de la región.

Por ejemplo, contar con un acueducto reparado y estable puede nutrir de agua el lago en donde podrían cultivar peces, o la construcción de un trapiche y la certificación del personal en Buenas Prácticas de Manufactura (BPM) les permitirá posicionarse como los principales productores de panela en la localidad. Estos dos proyectos de emprendimiento endógeno, en la medida en que sean factibles desde las lógicas técnicas, comerciales y económicas, al ser concebidos por la comunidad representan su identidad colectiva, aportan a procesos de participación comunitaria y no van en detrimento de las tradiciones, las costumbres y los demás elementos identitarios.

Finalmente, este estudio aporta al entendimiento y la reflexión del entramado histórico y social que han vivenciado las comunidades indígenas y que, a pesar de los cambios ideológicos, culturales y físicos se ha mantenido como parte fundamental de las raíces culturales del país, en la construcción de una identidad colectiva que permea a todos los individuos y que, por ser parte de una configuración multicultural, es necesario preservar.

En las comunidades indígenas del país se ha sufrido el abandono del Estado, la pérdida de sus tradiciones y valores ancestrales. Más allá de políticas asistencialistas, el Gobierno nacional debe promover mecanismos de participación ciudadana, programas de fortalecimiento de las capacidades asociativas y organizativas de las comunidades para que, de esta forma, generen espacios de discusión dirigidos al diagnóstico de las principales problemáticas, proyectos de desarrollo social y económico, así como una agenda de planificación que utilice como recurso base el territorio y los saberes ancestrales de las comunidades.
El conocimiento del territorio, la historia de la comunidad, la identidad cultural y la cosmogonía de las comunidades deben percibirse como el verdadero patrimonio de la sociedad, ya que permiten construir vínculos que sirven como base para generar procesos de desarrollo y organización comunitaria que posibilitan justicia y armonía entre las comunidades.

El estudio, en conjunto con las técnicas de investigación y acción participativa, puede aprovecharse en cualquier contexto. El reto implica un proceso de intervención con cada comunidad específica, a partir de la necesidad de hacer lecturas de contextos y procesos dialógicos de construcción colectiva. Desde el proceso de la investigación, los resultados presentados permiten proponer nuevos retos que pueden ser abordados por futuros procesos de indagación, así como se reconocen los límites propios del método y los determinados por el objeto de estudio.

\section{Referencias}

Altieri, M. A.; Toledo, V. (2011). La revolución agroecológica en América Latina. Revista El Otro Derecho, (42), 163-202. Recuperado de https://bit.ly/3is44c1

Aponte, M.; Álvarez, M. (2017). Cooperative entrepreneurship in Puerto Rico. International Journal of Entrepreneurship and Small Business, 32(3), 386-401. DOI: https://doi.org/10.1504/ IJESB.2017.087028

Arroyave-Puerta, A. M.; Marulanda-Valencia, F. A. (2019). Ecoemprendimiento, sostenibilidad y generación de valor. Revista Escuela de Administración de Negocios, (87), 155-172. DOI: https://doi.org/10.21158/01208160.n87.2019.2411

Ayres, R. U.; van den Bergh, J. C. J. M.; Gowdy, J. M. (2001). Strong versus weak sustainability: economics, natural sciences and <consilience>. Environmental Ethics, 23(1), 155-168. DOI: https://doi.org/10.5840/enviroethics200123225

Bargsted, A. (2013). El emprendimiento social desde una mirada psicosocial. Civilizar Ciencias Sociales y Humanas, 13(25), 121132. DOI: https://doi.org/10.22518/16578953.133

Berkes, F. y Adhikari, T. (2006). Development and conservation: indigenous businesses and the UNDP Equator Initiative. International Journal of Entrepreneurship and Small Business, 3(6), 671-690. DOI: https://doi.org/10.1504/ IJESB.2006.010920 
Chirinos-Araque, Y.; Pérez-Peralta, C. (2016). La responsabilidad social universitaria: emprendimiento sostenible como impacto de intervención en comunidades vulnerables. Revista Escuela de Administración de Negocios, (81), 91-110. DOI: https://doi. org/10.21158/01208160.n81.2016.1560

DANE (Departamento Administrativo Nacional de Estadística). (2005). Censo Nacional 2005. Recuperado de https://bit. ly/3yp9r0T

de Franco, A. (2003). A revoluçao do local: globalizaçao, glocalizaçao, localizaçao. San Pablo; Brasilia: Agencia de Educaçâo para Desenvolvimiento.

Fals-Borda, O. (2016). Reflexiones sobre la aplicación de los métodos de estudio en Colombia. Revista Direito $e$ Práxis, 7(12), 771-788. DOI: https://doi.org/10.12957/ dep.2016.21827

Feldman-Bianco, B.; Domenech, E.; Sanjurjo, L. (2020). Desplazamientos, desposesión y violencias. Historia $y$ Sociedad, (39), 7-23. DOI: https://dx.doi.org/10.15446/hys. $\mathrm{n} 39.88602$

Freire, P. (1974). Educación para el cambio social. Buenos Aires: Tierra Nueva.

Gallagher, B.; Lawrence, T. (2012). Entrepreneurship and indigenous identity: a study of identity work by indigenous entrepreneurs in British Columbia. International Journal of Entrepreneurship and Small Business, 17(4), 395-414. DOI: https://doi.org/10.1504/IJESB.2012.050162

Global Entrepreneurhip Monitor (2016). Emprendimiento social: reporte especial. Recuperado de https://bit.ly/3xt6tHG

Guáqueta, A.; Mantilla, G. (2005). Cómo manejar riesgos de seguridad y construir paz al mismo tiempo. Revista Escuela de Administración de Negocios, (55), 39-49. Recuperado de https://bit.ly/2Vse5Nh

Guzmán-Vásquez, A.; Trujillo-Dávila, M. A. (2008). Emprendimiento social-revisión de literatura. Estudios Gerenciales, 24(109), 105-126. DOI: https://doi. org/10.1016/S01235923(08)70055-X

Haesbaert, R. (2013). Del mito de la desterritorialización a la multiterritorialidad. Cultura y Representaciones Sociales, 8(15), $9-42$.

Hall, S.; du Gay, P. (2003). Cuestiones de identidad cultural. Buenos Aires: Amorrortu.

Heffes, A. (2012). La identidad revisitada. Identidades, 3(2), 8597. Recuperado de https://bit.ly/3lypDtn

Khan, I. M. (2014). Indigenous entrepreneurship among the Kalash. International Journal of Entrepreneurship and Small Business, 22(1), 115-134. DOI: https://doi.org/10.1504/ IJESB.2014.062134

Leff, E. (2016). Society, politics and nature. Knowledge for what sustainability? Novos Cadernos Naea, 19(1), 9-20. DOI: https://doi.org/10.5801/ncn.v19i2.3742
Maillat, D. (1998). Innovative milieux and new generations of regional policies. Entrepreneurship \& Regional Development, 10(1), 1-16. DOI: https://doi. org/10.1080/08985629800000001

Marques, C. S.; Santos, G.; Ratten, V.; Barros, A. B. (2019). Innovation as a booster of rural artisan entrepreneurship: a case study of black pottery. International Journal of Entrepreneurial Behavior and Research, 25(4), 753-772. DOI: https://doi.org/10.1108/IJEBR-02-2018-0104

Mtika, M. N. (2013) Economic security in an agrarian community. Africa Development, 38(1-2), 1-24. Recuperado de https://bit. ly/3ilWWxx

Murillo, J. (2018). Métodos de investigación en educación especial. Universidad Autónoma de Madrid.

Organización de las Naciones Unidas. (2015). Objetivos de Desarrollo Sostenible. Recuperado de https://bit.ly/3ilf1vK

Organización Nacional Indígena de Colombia. (2018). Embera chami. Recuperado de https://bit.ly/3xljumn

Organización Regional Indígena Valle del Cauca. (2012). Plan de Salvaguarda del Pueblo Emberá Chamí del Valle del Cauca. Recuperado de https://bit.ly/3fqAtNS

Ospina-Ramírez, D. A. (2018). Construcción de comunidades colaborativas desde el diseño y el emprendimiento endógeno. Revista Escuela de Administración de Negocios, (84), 63-77. DOI: https://doi.org/10.21158/01208160.n84.2018.1917

Peralta, J. D. (2019). Desarrollo regional y emprendimiento: evidencia para Colombia. Trimestre Económico, 86(342), 467490. DOI: https://doi.org/10.20430/ete.v86i342.656

Peredo, A; Anderson, R. B.; Galbraith., C.; Honing, B.; Dana, L. P. (2004). Towards a theory of indigenous entrepreneurship Benson Honig. International Journal of Entrepreneurship and Small Business, 1(1/2), 1-20. DOI: https://doi.org/10.1504/ IJESB.2004.005374

Polbitsyn, S. N. (2019). Russia’s rural entrepreneurial ecosystems. Economy of Region, 15(1), 298-308. DOI: https://doi. org/10.17059/2019-1-23

Polletta, F.; Jasper, J. M. (2001). Collective identity and social movements. Annual Review of Sociology, 27(1), 283-305. DOI: https://doi.org/10.1146/annurev.soc.27.1.283

Programa de las Naciones Unidas para el Desarrollo. (2014). Perfiles productivos territoriales: información de calidad para decisiones acertadas. Recuperado de https://bit.ly/3AaE0I3

Rist, S. (2005). Desarrollo endógeno como un proceso social. Revista Compás, (7), 26-29.

Rojas, J. (2012). Campesinos e indios en el suroccidente colombiano. Cali: Universidad del Valle.

Salazar, L. G. (2016). Conflicto armado y configuración territorial: elementos para la consolidación de la paz en Colombia. Bitácora Urbano Territorial, 26(2). DOI: https:// doi.org/10.15446/bitacora.v26n2.57605 
Sennett, R. (2007). Carne y piedra: el cuerpo y la sangre en la civilización occidental. Madrid: Alianza Editorial. Recuperado de https://bit.ly/3ypznJM

Shin, C. (2016). A conceptual approach to the relationships between the social economy, social welfare, and social innovation. Journal of Science and Technology Policy Management, 7(2), 154-172. DOI: https://doi.org/10.1108/ JSTPM-08-2015-0027

Spradley, J. (1979). La entrevista etnográfica. Nueva York: Holt, Rinehart y Winston.

Tapsell, P.; Woods, C. (2008). A spiral of innovation framework for social entrepreneurship: social innovation at the generational divide in an indigenous context. Emergence: Complexity and Organization, 10(3), 25-34.

Torres, A. (2013). El retorno a la comunidad. Problemas, debates $y$ desafíos de vivir juntos. Bogotá: CINDE; El Buho.

Vidal, T.; Pol, E. (2004). Un modelo de apropiación del espacio mediante ecuaciones estructurales. Medio Ambiente $y$ Comportamiento Humano, 5(1,2), 27-52.

Zapata, J. (2010). Espacio y territorio sagrado, lógica del ordenamiento territorial indígena. Bogotá: Universidad Nacional de Colombia. 